\title{
THREE NAVIGATION SYSTEMS WITH THREE TASKS: USING THE LANE-CHANGE TEST (LCT) TO ASSESS DISTRACTION DEMAND
}

\author{
Joanne L. Harbluk, Julia S. Mitroi, \& Peter C. Burns \\ Transport Canada \\ Ottawa, Ontario, Canada, \\ Email: joanne.harbluk@tc.gc.ca
}

\begin{abstract}
Summary: The Lane Change Test (ISO, 2008; Mattes, 2003) was used to assess distraction demand when drivers completed three typical navigation tasks (an easy navigation task, a point of interest task and a difficult navigation task) using three different navigation systems. In order for the LCT to be a useful procedure, it must distinguish good from poor navigation systems and acceptable from unacceptable tasks performed using those systems. The results provide some general support for the LCT as a sensitive measure of distraction. Some aspects of the results, however, called into question the adequacy of the LCT as a sufficient measure of distraction. In particular, the LCT was found to be insensitive to task demands arising from excessive task duration. Since risk exposure is a function of secondary task duration (as well as other factors such as intensity, frequency and timing), it is recommended that a measure of task duration be incorporated in the LCT procedure. When the MDEV was modified to incorporate task duration, the resulting measure (mean deviation per average task) reflected more adequately the interaction demands of the various navigation tasks.
\end{abstract}

\section{INTRODUCTION}

Appropriate protocols are required to assess the distraction potential of in-vehicle information and communication systems and the tasks that drivers perform using those systems. The goal of these assessment procedures is to provide drivers with safe equipment that is compatible with the task of driving, minimizing risk to ensure the safety of drivers and other road users. In-vehicle information and communication systems are designed to deliver information such as navigation guidance, road and traffic conditions, as well as entertainment and communication functions to drivers. System interface designs that require less demand of the driver are, by definition, safer systems.

Methodologies to assess driver interaction demand must be valid, objective and reliable. In addition, they must be low cost and practical if they are to be implemented. Ideally, a test or toolkit of tests that meet these requirements could be standardized and made widely available. These procedures would be useful in research, design, test and evaluation, compliance and product certification and would be of interest to researchers, manufacturers and regulators (Burns, Bengler \& Weir, 2009).

The Lane Change Test (LCT; ISO 2008; Mattes, 2003) is an assessment methodology that is standardized, low cost, easy to implement, and completed quickly. Drivers, seated at a computer, repeatedly perform lane changes prompted by road signs. The quality of these lane changes provides the metric for comparison. Lane change performance under dual task conditions 
(driving while using the system of interest) is evaluated against a normative model of single task performance. The extent to which the dual task condition results in increased distraction is reflected in impaired lateral control (MDEV). This MDEV measure is intended to be a combined measure of object and event detection, manoeuvring and lane keeping.

The purpose of the present study was to further explore the LCT with a particular focus on its utility to distinguish acceptable from unacceptable tasks performed using navigation systems. Three naviation systems were used. Two were readily available commerical aftermarket (nomad systems) and the third was an OEM system available on production vehicles. Participants performed three tasks using each of the three navigation systems. In order of increasing difficulty these were a low demand address task, a point of interest task and a high demand address task. Interactions with all three systems were carried out via visual-manual interaction. Speech-based interactions and cognitive demand were not the focus of this work (see Harbluk et al., 2007; Engström \& Markkula, 2007). Finally, because risk exposure is a function of secondary task duration (Shutko \& Tijerina, 2006; Wierwille \& Tijerina, 1998) as well as intensity, frequency and timing, the utility of a measure incorporating individual task durations (mean deviation per average task) was explored.

\section{METHOD}

\section{Participants}

The seventeen participants ranged in age from 21 to 59 ( 7 men; 10 women). All drivers were fully licensed and drove a minimum of $10 \mathrm{~K}$ kilometres annually.

\section{Equipment \& Materials}

LCT. A PC was used to present the Lane Change Test driving simulation on a video monitor. A Logitech MOMO Racing Force-Feedback Wheel with foot pedals was used for the control of the simulated vehicle (Figure 1). The test track was a $3 \mathrm{~km}$ straight lane road with 18 lane change

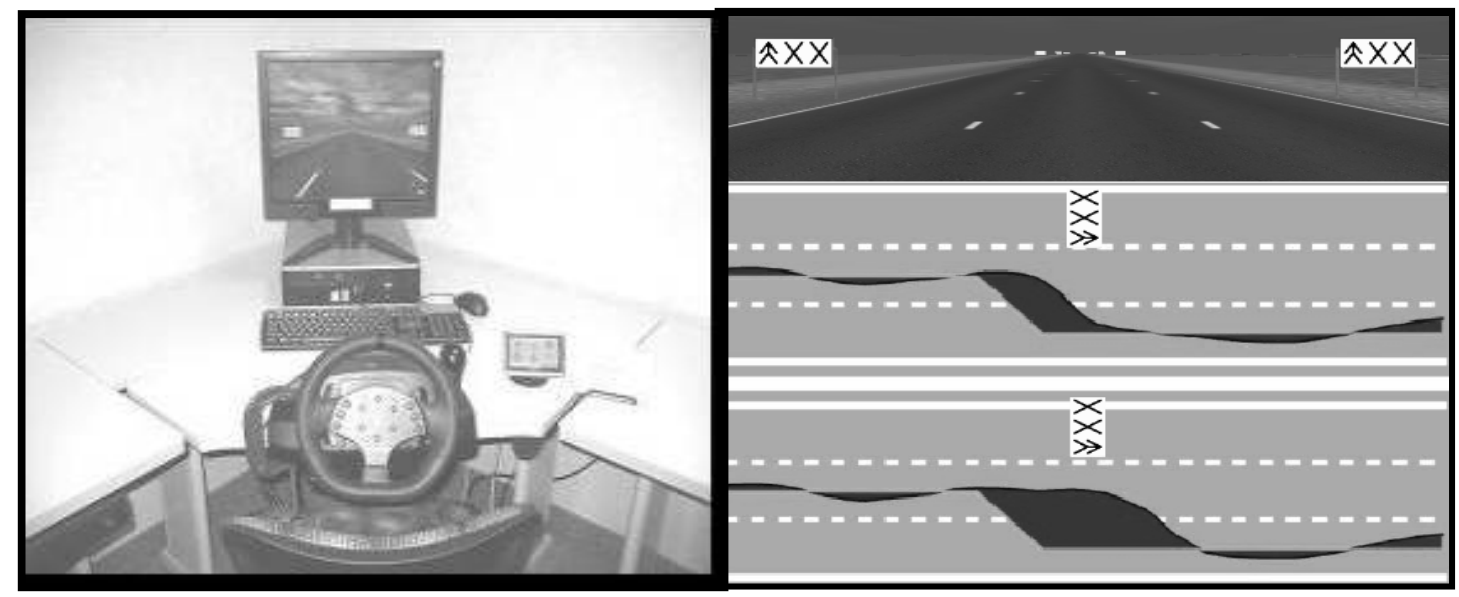

Figure 1. LCT Equipment Set Up

Figure 2. Image of LCT Monitor View and Illustrations of Performance 
signs. The mean distance between signs was $150 \mathrm{~m}$, resulting in a mean duration of 9 seconds between two lane changes since participants were required to perform lane-change manoeuvres while maintaining a constant speed of $60 \mathrm{~km} /$ hour (resulting in duration of approximately 180 seconds per track). The top frame of Figure 2 shows the driver's forward view of the simulated three-lane road scene with signs instructing the driver to change into the left lane. The middle frame illustrates the difference in area (black) between the normative path and actual path for a quick response (adapted from Mattes, 2003). The bottom frame illustrates performance during a slower lane-change response where the greater black area indicates poorer performance.

Navigation systems. The three navigation systems that were used are shown in Figure 3. Two (Systems A \& B) were popular, commercially available navigation systems with touch screen controls. The third (System C) was an OEM system removed from the vehicle for lab testing. Since the point of this research is not to assess the systems, but rather the LCT test itself, the systems are referred to as Systems A, B, \& C. All systems were operated in visual-manual mode. Drivers made selections using the touch-screens on System A and System B, and using the push buttons on System C.
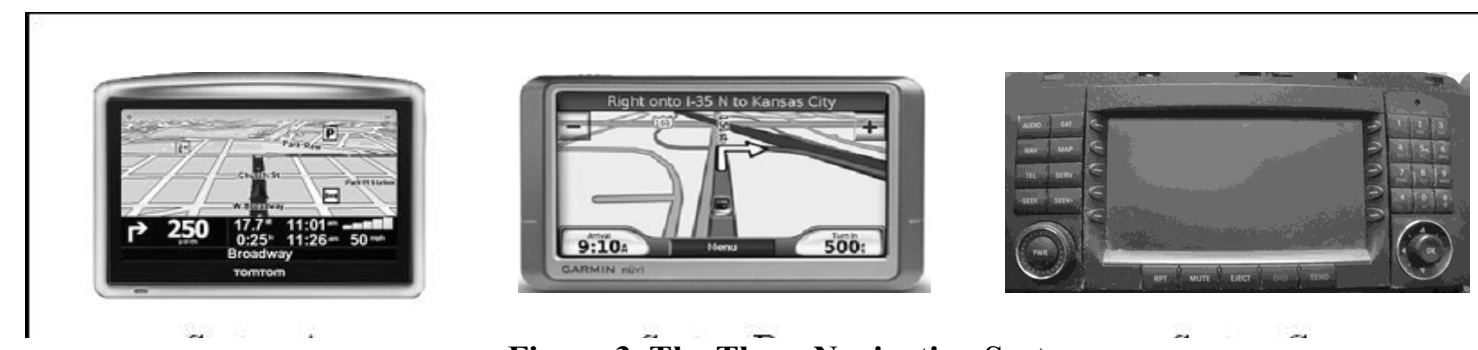

Figure 3. The Three Navigation Systems

Tasks. Three tasks, typical of real-world navigation tasks, were carried out using each of the three navigation systems. Tasks were designed to vary with respect to the required number of button presses and the time needed for completion. Table 1 presents the number of button presses and the mean time (s) required to complete each task when not driving. As can be seen in the table, the tasks varied considerably in terms of the interaction demand and time required for completion. In order of increasing difficulty these were a low demand address task (Address Lo), a point of interest (POI) task and a high demand address (Address Hi) task.

\section{Procedure}

Participants were instructed to perform their lane change manoeuvres as soon as they recognized the information on the sign. They were instructed to change lanes in a deliberate manner, as quickly and efficiently as possible. After receiving instructions, participants began the session with practice trials on the LCT as well as the secondary tasks. Participants then completed one 3minute run for each of the 9 experimental conditions created by the combinations of each of the three navigation systems with each of the three navigation tasks. Participants drove the track without performing a secondary task during the three baseline drives. The information for the secondary tasks was provided on $3 \times 5$ cards. The order of the experimental conditions was counterbalanced with the exception of the three baseline tasks, which took place at the beginning, middle and end of the experimental session. 
Table 1. Characteristics of the Three Navigation Tasks Performed Using the Three Navigation Systems: Number of Button Presses and Mean Time (s) Required for Completion

\begin{tabular}{|l|l|r|r|}
\hline System & Task & Button Presses & Static Time \\
\hline A & Address Lo & 4 & 2.059 \\
\hline & POI & 7.4 & 14.529 \\
\hline & Address High & 13.5 & 16.294 \\
\hline B & Address Lo & 3 & 2.353 \\
\hline & POI & 6.4 & 12.706 \\
\hline & Address High & 19 & 26.882 \\
\hline C & Address Lo & 3 & 4.176 \\
\hline & POI & 16.4 & 26.882 \\
\hline & Address High & 38.3 & 63.529 \\
\hline
\end{tabular}

Participants were reminded that their primary task was to drive as safely as possible, as they would in the real world, and were asked to perform secondary tasks as quickly and accurately as possible. There was no surrounding traffic in the scenario.

\section{RESULTS}

Mean deviation in lane change path $(M D E V)$. This measure represents the mean deviation between the position of the normative model and the actual driven course. Following the procedure recommended for outliers, MDEVs exceeding 2SD from the mean for that condition (.04 of the data) were replaced with the value of 2SD (ISO, 2008). The MDEV data are presented in Figure 4. The same pattern of results was found across all three navigation systems. The LCT MDEV measure clearly discriminated when participants were performing a secondary task using the navigation systems. (ANOVAs for System A: $F_{(3,48)}=39.59, p<.001$; System B: $F_{(3,48)}=$

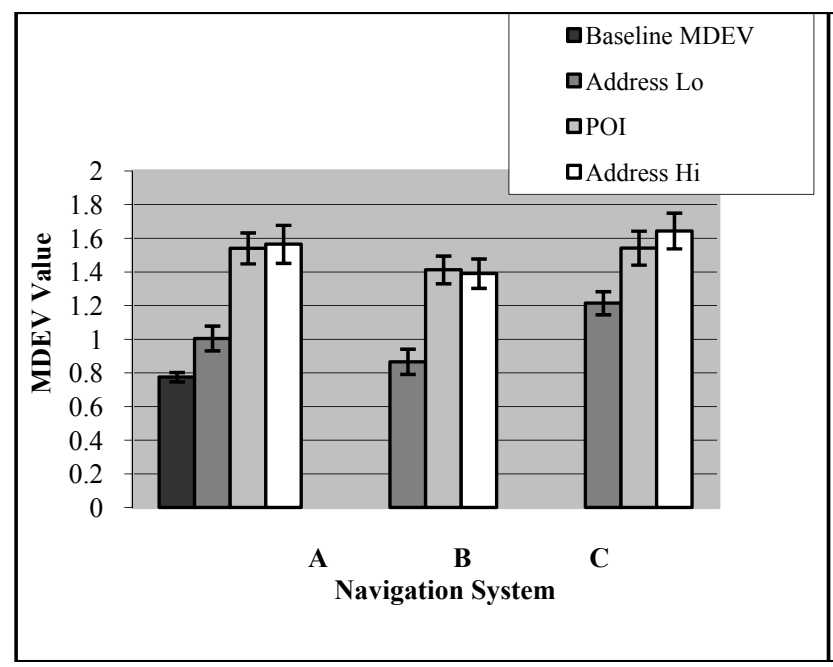

Figure 4. MDEV Values (+/-SE) for the Three Tasks Using the Three Navigation Systems

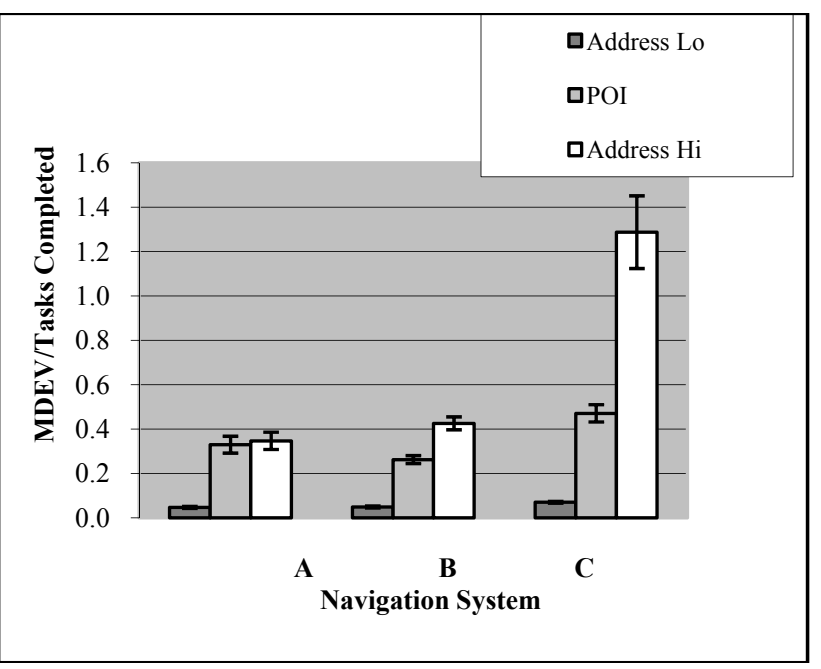

Figure 5. MDEV Values (+/-SE) Divided by Number of Tasks Completed During a 3 Minute Run (MDEV task) 
40.73, $\mathrm{p}<.001$; for System $\mathrm{C}: \mathrm{F}_{(3,48)}=46.93, \mathrm{p}<.001$; all post hoc comparisons with baseline were significant at $\mathrm{p}<.05$ ). Finally, the MDEV values for the POI and Address Hi tasks were significantly greater than those for the Address Lo task (all ps<.05).

The MDEV measure, however, did not indicate differences between the POI task and the Address Hi task (ps>.05) as expected. This lack of MDEV discrimination between POI \& Address Hi Tasks is troublesome. For Systems B and C, the Address Hi Tasks require at least two times the number of button presses and the time taken to complete the Address Hi tasks is double that required for the POI tasks.

Task Duration. This measure represents the mean time required, in seconds, for one completion of a particular secondary task while driving the LCT (Table 2). It is important to consider the relationship between the structure of the LCT test and task duration. The LCT is designed as a fixed 3-minute drive with the consequence that a shorter task must be repeated many times to "fill" the 3 minutes whereas a longer task might be completed only once or twice during that period. Thus, when comparisons are made across tasks, the shorter task and longer tasks are treated as though as they are of equivalent duration. There is no "benefit" accorded a short task even though we know that a shorter task would result in less risk since the driver's attention is drawn from the driving task for less time. A concrete example from city driving demonstrates why task duration is important. A driver may pass through five intersections while performing a long task, but only one intersection while performing a short task.

Table 2. MDEV Values, Task Completion Durations, and Work Load Ratings During the LCT

\begin{tabular}{|l|l|r|r|r|}
\hline System & Task & \multicolumn{1}{|l|}{ MDEV } & \multicolumn{1}{l|}{ Duration } & Work Load \\
\hline & Baseline & 0.77 & & 2.18 \\
\hline $\mathbf{A}$ & Address Lo & 1.00 & 3.28 & 4.15 \\
\hline & POI & 1.54 & 25.42 & 7.88 \\
\hline & Address High & 1.56 & 26.66 & 8.25 \\
\hline $\mathbf{B}$ & Address Lo & 0.87 & 3.40 & 4.29 \\
\hline & POI & 1.41 & 21.95 & 7.03 \\
\hline & Address High & 1.39 & 42.15 & 7.35 \\
\hline $\mathbf{C}$ & Address Lo & 1.21 & 6.18 & 6.62 \\
\hline & POI & 1.54 & 41.76 & 8.84 \\
\hline & Address High & 1.64 & 91.45 & 9.47 \\
\hline
\end{tabular}

As can be seen in Table 2, navigation tasks performed during the LCT differ considerably as to the amount of time required for a single completion, from $3.28 \mathrm{~s}$ to $91.45 \mathrm{~s}$, representing an important dimension of interaction demand. Table 2 displays the MDEV values as well as task durations for the various tasks completed during the LCT. Of particular concern are the MDEVs and durations for the POI and the Address Hi tasks for systems B and C. As can be seen in Table 2, MDEV values for the two tasks are similar for each of the navigation systems. Yet, no consideration is given to the task durations for completion, which are twice as long for the Address Hi task compared with the POI task for both of these systems. 
Both the MDEV values and duration were relatively low for the Address Lo tasks, the easiest on all three navigation systems.

Mean deviation per average task ( $M D E V_{\text {task). }}$. This measure represents the mean deviation (MDEV) divided by the number of tasks completed during the 3 minute run. Given the large discrepancies in task duration and the fixed LCT duration of 3 minutes, we adopted a procedure whereby the MDEV value was divided by the number of task completions during the run. This new measure, $\mathrm{MDEV}_{\text {task, }}$ incorporates the MDEV as described above, but also takes into account task duration. As can be seen in Figure 5, MDEV task provided a better "fit" to the driver interaction demands of the navigation system tasks. Comparisons using ANOVA were made for the three tasks for each of the three navigation systems. All ANOVAs were significant across the tasks for each of the three navigations systems. For Systems $B\left(F_{(2,32)}=118.46, p<.001\right)$ and $C$ $\left(\mathrm{F}_{(2,32)}=44.05, \mathrm{p}<.001\right), \mathrm{MDEV}_{\text {task }}$ indicated significant differences reflecting the order of task demand for Address Lo, POI and Address HI respectively (all ps $<.05)$. For System A $\left(\mathrm{F}_{(2,32)}=\right.$ 45.08, $\mathrm{p}<.001)$, the Address Lo was less than the POI and Address Hi which did not differ significantly from each other.

Workload Ratings. Ratings of subjective workload (10 point scale; $1=$ low, $10=$ high) provided by the participants (see Table 2) were consistent with the task difficulty characteristics of the tasks as outlined in Table 1.

\section{DISCUSSION}

In this paper, LCT performance (MDEV) for three tasks using three navigation systems was examined. For all three systems MDEV was greater when a task was being performed while driving compared to baseline driving. In addition, MDEV discriminated Address Lo tasks (relatively easy tasks) from the more difficult POI and Address Hi tasks. In contrast, the inability of MDEV to discriminate between POI and Address Hi Tasks was problematic. The Address Hi Tasks for Systems B and C have approximately twice the number of button presses and take twice as long to complete as the POI tasks, yet the MDEV measure does not reflect these additional demands.

We propose that the LCT procedure would benefit from inclusion of a measure of task duration such as Mean Deviation per Task $\left(\mathrm{MDEV}_{\text {task }}\right)$. This recommendation is based on:

1) The need to consider task duration in risk assessment. The duration required to complete a specific task is an important, safety-relevant measure since it indicates the amount of time the driver's attention will be drawn away from the primary task of driving.

2) The observation that tasks differ considerably in duration and that this should be taken into consideration in the assessment of task demand. Mean durations for tasks in the present study varied widely from 3.28 to 91.45 seconds. Shorter tasks will tend to be safer. Given the long interaction times and high MDEV values, we would suggest that tasks such as the Address Hi tasks not be allowed while driving a vehicle. 
3) The inadequacy of MDEV to account for appreciable differences in task duration/interaction demand as shown in the results of this study.

In summary, the purpose of distraction assessment procedures such as the LCT is to provide drivers with safe equipment that is compatible with the task of driving. No single methodology is likely to be sufficient for this task (Angell et al., 2006). A "toolkit" comprised of a variety of distraction assessment methodologies, such as eye glance behaviour and LCT, will likely be required to provide a complete assessment of workload and distraction. With respect to the LCT, MDEV captures some of the dimensions of concern, but additional measures such as the $\mathrm{MDEV}_{\text {task }}$ could increase the utility of the LCT procedure.

\section{REFERENCES}

Angell, L., Auflick, J., Austria, P.A., Kochhar, D., Tijerina, L., Biever, W., Diptiman, T., Hogsett, T.J., \& Kiger, S. (2006). CAMP Driver Metrics Workload Project Task 2 Final Report, DOT HS 810 635. Washington, DC.

Burns, P.C., Bengler, K., \& Weir, D. (2009). User Needs and Uses of Driver Metrics: Panel Discussion. Driver Metrics Workshop, June 2, 2008, San Antonio Texas.

Engström, J., \& Markkula, G. (2007). Effects of visual and cognitive distraction on lane change test performance. Proceedings of the Fourth International Driving Symposium on Human Factors in Driver Assessment, Training and Vehicle Design, Stevenson, Washington, 199 205.

Harbluk, J.L., Burns, P.C., Lochner, M., \& Trbovich, P.L. (2007). Using the Lane Change Test to Assess Distraction: Tests of Visual-Manual and Speech-Based Operation of Navigation System Interfaces. Proceedings of the Fourth International Driving Symposium on Human Factors in Driver Assessment, Training and Vehicle Design, Stevenson, Washington, 16 22.

ISO. (2008). Road vehicles - Ergonomic aspects of transport information and control systems Simulated lane change test to assess driver distraction. ISO/TC 22/SC 13 N WG8 N579, DIS 26022.

Mattes, S. (2003). The lane change task as a tool for driver distraction evaluation. In H. Strasser, H. Rausch \& H. Bubb (Eds.), Quality of work and products in enterprises of the future. Stuttgart: Ergonomia Verlag.

Shutko, J. \& Tijerina, L. (2006). Eye Glance Behavior and Lane Exceedences During Driver Distraction. Driver Metrics Workshop, October 2-3, 2006 Ottawa, Ontario. http://ppc.uiowa.edu/drivermetricsworkshop/

Wierwille, W.W. \& Tijerina, L. (1998). Modeling the relationship between driver in-vehicle visual demands and accident occurrence. In Vision in Vehicles VI, Gale, A.G., Brown, I.D., Haslegrave, C.M., and Taylor, S.P. (Eds.), North-Holland, Amsterdam, pp. $233-244$. 